\title{
Fibrin and thrombosis in the central nervous system in children with particular reference to congenital hydrocephalus
}

\author{
JOHN L. EMERY \\ From the Department of Pathology, The Children's Hospital, Sheffield
}

While rare metabolic and degenerative diseases of the central nervous system in children are reported at great length, the much commoner diseases relating to vascular incidents are rarely discussed.

Only those aspects of disease of the central nervous system related to thrombosis and the deposition of fibrin will be discussed here; diseases related to general vasculitis and aneurysm are omitted. The conditions discussed here fall into two main categories: first, the presence of thrombosis in small blood vessels or in the large superficial veins draining the brain, and second, the deposition of fibrin either in the ventricles or on the membranes overlying the brain in association with thrombotic lesions, haemorrhage, or infiltration.

\section{PRIMARY THROMBOSES}

Thrombosis of small blood vessels, particularly of veins, forms an important part of the general pathology of infants. These lesions are easily diagnosed and recorded in viscera such as the kidneys but their importance in the brain is often overlooked. Thrombi in the brain can be found in two main forms, the first being massive thrombi in either the longitudinal sinus, the lateral sinus, or more commonly, one or other of the large veins coursing into the longitudinal sinus from the surface of the hemispheres. These thrombi are usually associated with cerebral symptoms, usually severe convulsions, and are rarely an incidental finding at necropsy. The more common finding when routine microscopy of the brain is done on all children is the finding of unequivocal ante-mortem thrombi in small vessels, particularly in the small vessels of the thalamus and of the cortex. Thrombi in the medulla and in the region of the olives are, in my experience, less common. These minute thrombi usually contain coagulated fibrin and a relatively few immeshed red blood cells. Occasionally many platelets appear to be incorporated but these are scanty and a simple fibrin clot is rarely seen. $\vec{v}$ Surrounding the small veins there are often clear vacuolated spaces but there is no evidence of inflammation of the vein wall or of reaction in the surrounding glia. Occasionally there are small local haemorrhages. There are three clinical states in which thrombi are most likely to be encountered.

SEVERE DEHYDRATION AND INANITION This situationo is liable to occur associated with vomiting or ${ }^{+}$ diarrhoea. There is frequently massive thrombosis, 0 often involving the longitudinal sinus and wells known in the past as 'marantic thrombosis'. The second condition is liable to occur in youngo infants associated with states of hypoxia. These can尺 be associated with birth or, what is probably most commonly and most frequently overlooked, with3 hypoxia related to acute tracheo-bronchitis. The third group is associated with peripheral vascular: failure from many causes, including post-operative shock and septicaemia.

The clinical effects associated with massive thrombosis are usually gross convulsive states. leading to death. Associated with the microscopic clots, symptoms may be minimal. Many instances of so-called febrile convulsions are probably associated? with these lesions. Such clots are frequently found in children found unexpectedly dead who have inhaled, and it is not uncommon for a child to die unexpect-N edly in this way. Such children also frequently show the presence of agonal intussusceptions in the smallo intestine, suggesting that there has been a terminal $\omega$ derangement of the function of the nervous system?

\section{HAEMORRHAGES}

INTRAVENTRICULAR HAEMORRHAGES Gross haemor- $-\frac{T}{0}$ rhage with clotted blood filling the ventricles, $\frac{\overrightarrow{\mathbb{D}}}{\mathrm{D}}$ particularly in the lateral and third ventricles, is a? not uncommon association with the newborn state. ㅇ

The sites of these haemorrhages are not always 
identifiable but the frequent finding of small areas of thrombosis on the choroid plexus in children who have died from haemorrhages elsewhere in the body suggests that the site of origin of the intracranial haemorrhage is probably the choroid plexus. The other most common site is in the highly vascular mass of primitive neuroblasts lying beneath the lining of the lateral ventricles in the premature child.

It is commonly believed that hydrocephalus due to blocked aqueducts is often due to scarring after subclinical intraventricular haemorrhage but in our experience this has been extremely rare, and, while we have found thrombi in these sites, I personally do not believe that clots within the ventricular system are of any great importance in the aetiology of hydrocephalus.

SUBDURAL HAEMORRHAGES Subdural haemorrhages are extremely common and probably an almost invariable concomitant of normal birth. Haemorrhages over the surface of the hemispheres are a very frequent incidental finding in the newborn period and evidence of old haemorrhages common findings in children dying in infancy. The majority of such haemorrhages appear to be completely asymptomatic and are of no clinical importance. The blood forms in these sites what appear to be simple clots which are eventually completely eliminated.

The only haemorrhages that appear to persist and produce any symptoms are those which occur over the upper and lateral surfaces of the cerebral hemispheres. In these sites, the haemorrhages liquefy and form the so-called chronic subdural haematoma. In our experience, every such case that has come to necropsy has shown the presence of thrombi of long standing in some of the traversing blood vessels. Since the essential difference between these persisting cystic cavities and the apparent dissolution of clots elsewhere is the presence of the traversing vessel it seems likely that the thrombosed traversing vessels are the essential factor in maintaining the chronicity of these subdural effusions.

\section{TRAUMA}

We have been able to study the effects of simple trauma from such procedures as ventricular puncture at considerable length in children with congenital hydrocephalus, and in none of these cases have we seen fibrin deposited in the injured area in the absence of chronic infection or gross haemorrhage. The tip of the Spitz-Holter catheter penetrating the side of the brain quite frequently impinges on the medial surface of the hemispheres and this can cause considerable damage to this area without the finding of any local tissue reaction.

\section{MENINGITIS AND ENCEPHALITIS}

It is doubtful if meningitis is ever a primary disease in a child. Since so many ill children show the presence of small haemorrhages or small thrombi, the impression gained is that a child with a septicaemia who has any such lesions is liable to develop infection at these sites spreading to the rest of the meninges.

The presence of pus itself, either within the ventricles or surrounding the brain, appears to have very little effect either on the circulation of the cerebrospinal fluid or on the function of the brain or indeed often on the general health of the child. The symptoms and complications are, in our experience, invariably associated with vascular lesions, either vasculitis affecting the small arteries or thrombosis of the veins. This is particularly notable in tuberculous meningitis in which the complications and final sequelae are directly related to the vascular damage. In this type of meningitis, masses of fibrin can be seen spreading outside the small arteries into the subarachnoid space and these are associated with inflammatory change in the walls of the blood vessels (Fig. 1). By the time any obstructive lesion has been found in the ventricular system, the obstructive structure itself has, in our experience, always been collagenous and not fibrinous.

There is often a complete dissociation between ventriculitis and meningitis and this is particularly seen in children with hydrocephalus associated with the Arnold-Chiari deformity. In these children, meningitis is virtually non-existent even in the presence of gross ventriculitis. In the cases of chronic ventriculitis often associated with $E$. coli and Pseudomonas pyocyanea, the ventricles are lined by a layer of coagulated fibrin and degenerate leucocytes but the presence of this membrane is always associated with areas of thrombosis and haemorrhage in the underlying brain tissue.

The ventriculitis and the 'fibrin' membrane in cases showing minimal ventriculitis is frequently localized to the choroid plexuses, these often being completely surrounded by a greyish zone of fibrinous tissue. The underlying choroid plexus in such cases invariably shows thrombosis and inflammatory change in the vessel wall. The 'fibrin' membrane referred to here contains thick masses of coagulated material and it seems that the presence of ventriculitis is invariably associated with vascular lesions either of the choroid plexus or of the brain tissue and follows a lesion of the choroid plexus.

In cases of ventriculitis that have been treated by the instillation of corticosteroids, the fibrin zone, previously described, is absent and the ventricle is lined by an irregular mass of degenerated nerve cells 


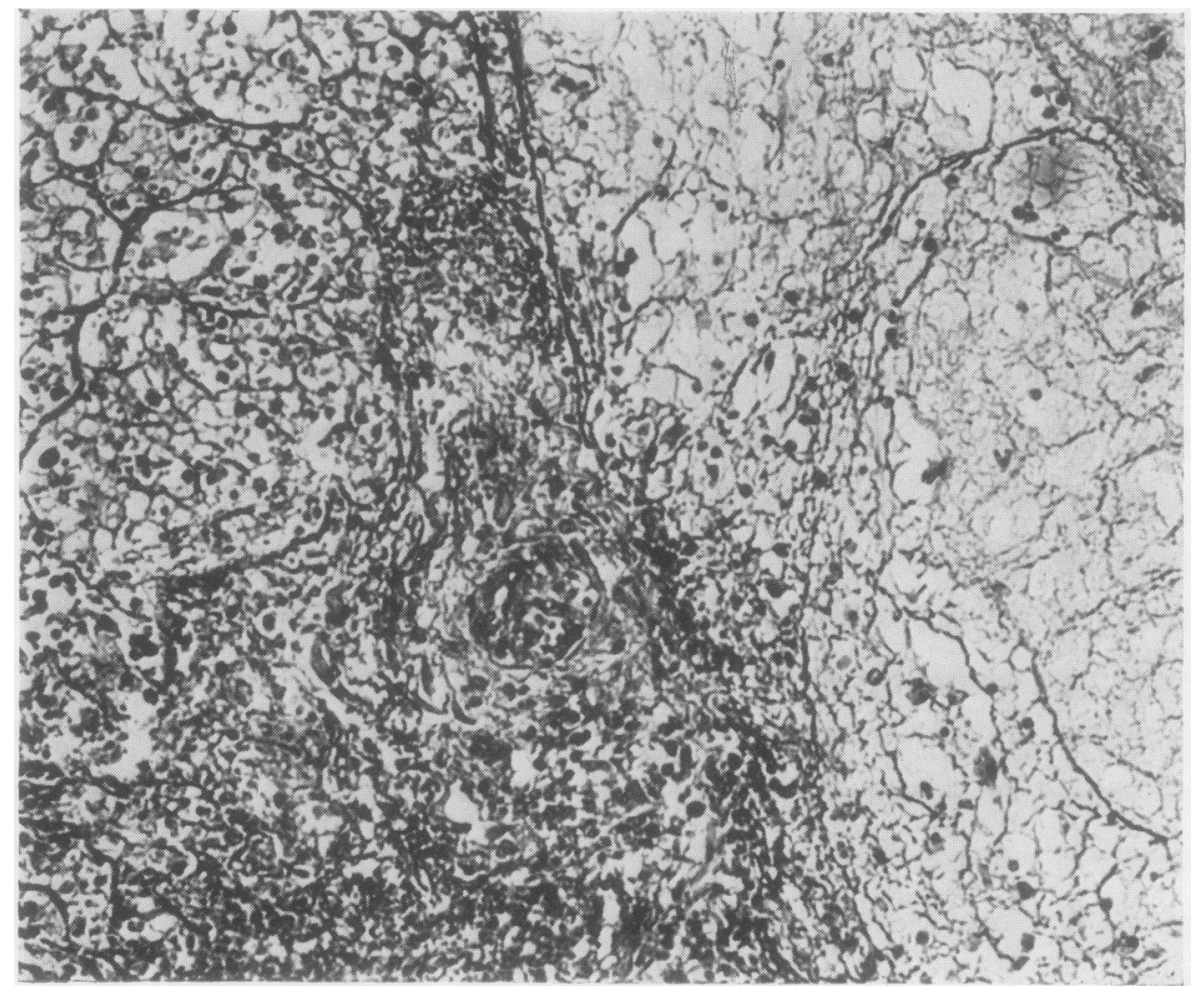

FIG. 1. Photographo of a section from below the base of the brain of a child with tuberculous meningitis. Near the centre of the picture is a small blood vessel almost completely occluded by swelling of the intimal cells. The surrounding subarachnoid space contains an extensive network of fibrin. (Masson $\times 120$ ).

and leucocytes, there being no definite line of demarcation between the ventricular cavity and the brain tissue.

\section{CHANGES ASSOCIATED WITH HYDROCEPHALUS TREATED WITH THE SPITZ-HOLTER VALVE}

The cerebral end of the Spitz-Holter valve system that lies within the brain appears to cause virtually no reaction, and except in the presence of ventriculitis we have not seen the upper end of the valve obstructed by any simple fibrin mass. When a membrane is found it appears to consist of an admixture of coagulated fibrin, fat, and degenerated cell bodies and in no way resembles the fibrin as seen in an artificial clot. In the valvular system itself, we have never found fibrin alone. When the valves are infected there are simply irregular masses of organisms. The lining of the valve system appears to be particularly non-reactive and the surface accepts only a few granules of amorphous protein material none of which stain for fibrin.

The lower end of the valve, whether lying within the abdominal cavity, within the right auricle, or within any other of the body cavities in the majority of children produces no reaction whatsoever, and we have seen tubes that have lain within the right auricle of the heart for two years in which not a윽 vestige of fibrin was attached to the surface. There is, however, invariably a collar of fibrous tissue aroundo the tube where it lies within the superior vena cava.

On the other hand, there is a group of children, probably representing between $5 \%$ and $10 \%$, in $\frac{0}{3}$ whom there is persistent reaction between the cerebrospinal fluid and the tissues, whether this be을 the peritoneal cavity or the freely-flowing blood in the heart. Such children develop irregular clots on the 을 ends of the intracardiac catheter (Fig. 2), and $D$ fibrous sacs around the end of the catheter within the peritoneal cavity. Thus, there appear to be two N types of children, those who react to their own cerebrospinal fluid, and the other group, the non- $N$ reactors. The 'reactors' are an important clinical $\omega$ group and in them the cerebrospinal fluid appears to incite the deposition of what is probably fibrin or ao fibrin precursor. When this reaction occurs within $\bar{\varnothing}$ the heart, massive thrombi form in the right auricle? which can cause obstruction to the tricuspid valve or ${ }^{\circ}$ death from gross pulmonary embolism. Smaller emboli cause incidents of what is frequently called $\stackrel{D}{\stackrel{D}{\infty}}$ 'acute pneumonia', or if the emboli are multiple $\stackrel{\mathbb{\perp}}{\circ}$ and frequent, the picture of haemolytic anaemia and pulmonary haemosiderosis. 


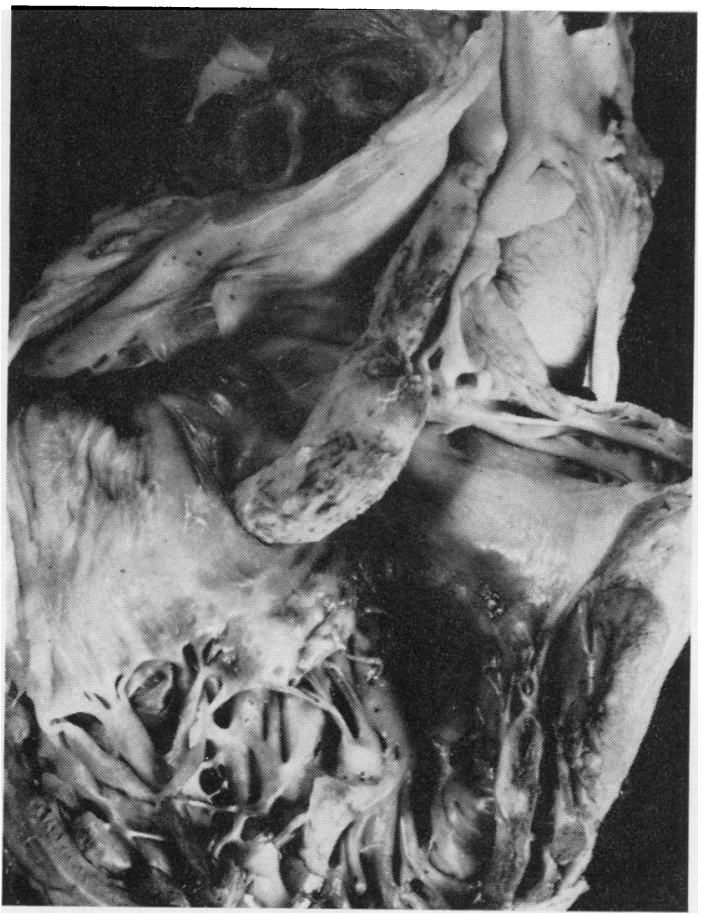

FIG. 2. View looking into the right auricle from a child with hydrocephalus treated with a Spitz-Holter valve system between the cerebral ventricle and the heart. The lower end of the shunt catheter is surrounded by an irregular mass of fibrin extending down to the tricuspid valve. The catheter itself only extends a short distance into the top end of the clot.

\section{SUMMARY}

Thrombosis and vasculitis of the brain are important primary conditions in children and are often the cause of serious secondary intracerebral conditions.

When complications due to the deposition of fibrin and inflammation occur, obstructive lesions are usually due to the rapid conversion of the fibrinous exudate to collagen, taking place with much greater rapidity in the child than in the adult.

There appear to be two types of children. Most children are 'non-reactors', freely allowing the presence of their own cerebrospinal fluid within their tissue spaces, the other group ('reactors') responding to the presence of cerebrospinal fluid by the deposition of fibrinoid protein which is rapidly converted to collagen. The cause of this process is obscure and requires active investigation.

MR. NIXON described eight cases in which ventriculitis followed surgery. In seven of these the cerebrospinal fluid was sterilized by giving fibrinolytic agents together with antibiotics, both intraventricularly as well as systemically. Local use of streptokinase or urokinase was not associated with any ill effects. He suggested that fibrinolytic agents might be used prophylactically in operations for insertion of the Spitz-Holter valve, in which fibrin deposition in relation to the prosthesis might jeopardise the result. Some patients, 'reactors', seemed particularly prone to this complication, though the underlying cause had not yet been clarified.

DR. CROME mentioned endocarditis as a complication of the Spitz-Holter valve. Nevertheless he was puzzled not so much that endocarditis occurred as that in most cases it did not. Like Dr. Emery he was satisfied that thrombotic phenonema in small blood vessels of the brain were of pathogenic importance, but that clotting of blood as a cause of hydrocephalus had been exaggerated. He had only seen one case in which obstruction of the aqueduct of Sylvius by clot might have been the cause of obstructive hydrocephalus. In his experience clotting of the proteinrich exudate in septic infections would account for some cases of obstruction. Ventriculitis as a complication of surgical procedures for hydrocephalus was in his experience associated with what he believed to be a primary choroiditis. He felt that it was important to differentiate between laboratory cure and clinical cure, in that although the cerebrospinal fluid might be rendered sterile and remain so after death, there could be quite profound changes in the ventricles consisting of deposition of collagen involving the choroid plexus.

DR. BRUCE presented the clinical data from Sheffield with which Dr. Emery's work was connected. Since 1958, 381 children had had Spitz-Holter valves inserted. The overall mortality rate during five years had been $26 \%$. The number of children not requiring any further surgery was 264 , that is, $68 \%$ of the total. Two hundred and twenty-four children had had the shunt for more than a year, and of these $11 \%$ were dead, but $60 \%$ had required no further operative procedure. Thirty-six per cent had needed one to four further operative procedures. Six children, who would fall into Dr. Emery's classification of 'reactors', had required up to nine revisions. The practice at Sheffield had been to start with a ventriculoatrial shunt. If this failed the shunt was converted into a ventriculo-peritoneal one.

DR. STEWART asked if the protein content of the cerebrospinal fluid of these cases was more or less normal.

DR. BRUCE replied that the protein level was certainly below $60 \mathrm{mg} . \%$ in all before the shunt was put in.

MR. FORREST stated that it was his practice to put the lower end of the valve into the jugular vein. He had put valves into nine patients in whom the protein content of the cerebrospinal fluid was over $100 \mathrm{mg} . \%$ and most of these had protein levels in the region of 1,200 to $1,500 \mathrm{mg} . \%$, but in only one was there any suggestion of blockage of the valve. He felt that too much time was wasted in attempts to reduce the protein level in the cerebrospinal fluid before inserting the valve.

DR. FEARNLEY AND DR. LACK thought that analysis of the obstructions found at the bottom end of Spitz-Holter valves would yield important information. The nature of these obstructions was not entirely clear: platelets, 
fibrin and deoxyribose nucleic acid might all be playing a part and might yield to therapeutic measures.

PROFESSOR GILLINGHAM, referring to Mr. Nixon's cases, quoted the case of a child with an astrocytoma of the pons filling the fourth ventricle. Successful subtotal removal was followed two years later by progressive hydrocephalus from widespread obstruction of the subarachnoid space. It was thought to have arisen from continued protein transudate from remaining tumour fragments, but in fact the cerebrospinal fluid protein level at that time was between 60 and $70 \mathrm{mg} . \%$. A peritoneal shunt operation was performed from the lumbar subarachnoid space but failed after three weeks because of obstruction due to the lining of a small cystic cavity around the tube within the peritoneal cavity over the anterior surface of the liver. The distal end of the tube was withdrawn from this cavity and was seen to be patent, unobstructed by fibrinous clot. Cerebrospinal $\stackrel{\stackrel{2}{二}}{\frac{0}{2}}$

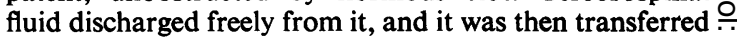
to the pleural cavity. Almost at once it was followed by a large accumulation of cerebrospinal fluid in the pleural $\stackrel{\overrightarrow{+}}{+}$ cavity which ultimately led to severe and incapacitating dyspnoea. Repeated aspirations of the pleural cavity were necessary. The fluid was of a greenish colour and $\bar{D}$ slightly opalescent. It contained $4.6 \mathrm{~g} . \%$ protein. Hyalase, $\overparen{\Phi}$ $1 \mathrm{ml}$., was injected into the pleural cavity after aspiration of the fluid on one occasion and thereafter no further $\infty$ aspiration was required. $X$-ray studies of the chest showed $\overrightarrow{0}$ that the fluid disappeared completely over three days and the child improved immediately and has remained well $\vec{\omega}$ over the subsequent 11 years.

MR. NIXON replied that he had tried the effect of hyalase $\frac{9}{0}$ in the pleural cavity in a patient who developed a big effusion after a pleural shunt but without improvement. 